was performed on Feb. 14th, 1896. The abdomen was opened by an incision through the substance of the left rectus muscle. The usual sausage-shaped tumour was found, tense, and freely adherent. The adhesions bled freely, as they were separated, and the cyst burst, discharging very fotid pus into the sponges placed in the pelvis. After the removal of the left appendage the right was found in the same condition and was remored. It also burst and discharged similar fotid pus. As contamination of the peritoneum could not be avoided, it was well sluiced with 1 in 10,000 sublimate solution, and carefully cleansed, a glass drainage tube being lef: after securing all bleeding points. The patient bore the operation well. An hour afterwards three ounces of blood were drawn off through the tube, but after that nothing of consequence came away. Next morning she was apparently going on well, but twentyfour hours after the operation she suddenly collapsed, with an almost imperceptible pulse; the face was pallid and shrunken, and there was every appearance of impending death. Two drachms of an almost colourless fluid were drawn off through the tube, thus proving the absence of internal bleeding. The median basilic vein was promptly opened, and three pints of normal saline solution at a temperature of $112^{\circ} \mathrm{F}$. were slowly thrown in by gravitation. The effect was immediate, the pulse became steady and easily counted (150). A quarter of an hour after the injection she vomited some bile-stained fluid; this was followed in half an hour by a well-marked rigor, with sweating. The temperature was $102^{\circ} \mathrm{F}$., rising in a short time to $104^{\circ}$. Four hours afterwards the pulse had fallen to 115 , the patient had greatly improved, and she said that new life had been put into her. The improvement continued, but her recovery was retarded by the abundant discharge of pus of the same foetid character as that in the tubes at the operation. The pelvis was repeatedly washed out daily with Condy's fluid, but for some days without effect, when diluted sulphurous acid was tried, yet still without effect; but the foetor quickly yielded to crystallised ioloform thrown down the tube, which was soon afterwards withdrawn, as the discharge ceased, and recovery went on slowly but without further drawback.

It is worth noting that the loss of blood at the operation was not excessive, and did not continue long afterwards. The collapse on the following day seems to have been due to loss of vaso-motor influence following on the abdominal operation. The patient was, in fact, bleeding to death into the vessels of her own abdominal viscera. The saline injection kept the circulation going until it was able to draw upon the blood temporarily lying in those vessels, and restore it to the general circulation.

The injection of saline solution has been resorted to rather freely at the Newcastle Infirmary, and in most cases with admirable effect. The two following cases are examples taken from several others in my own wards.

CASE 2 -A woman was admitted with a large collection of pus in the knee-joint, following white leg after confinement. The joint was incised in two or three places, washed out, and drained, with relief, but a day or two afterwards she was apparently sinking. After injection she quickly rallied, and for a time improved, but the joint was disorganised and she began to lose ground. Amputation high in the thigh was performed, when she nearly died on the table; bat injection of the saline solution, which had been got ready in anticipation, brought her round, and she rapidly recovered.

CASE 3.-A man aged fifty years had his hand caught in machinery and was carried five times round a revolving shaft. He was admitted with fracture of several ribs on the left side, fracture of the sternum, and fracture of nearly every rib on the right side, so that the right side of the chest sank in with each inspiration. There was extensive subcutaneous emphysema on the right side, and the right arm was smashed, so as to require amputation through the humeras. There was extreme pallor and depression, but the pulse was fairly good, so I ventured to amputate; but we had saline solution ready, and before closing the stump forty ounces were poured into the open end of one of the veins, with instant good effect. No bandage or plaster was applied to the chest. A month after the accident he was nearly well, and ultimately recovered perfectly.

I am indebted for the notes of the cases to my late house surgeon, Dr. R. W. Morgan.

Newcastle-on-Tyne.
THE THERAPEUTICAL ASPECTS OF TALKING, SHOUTING, SINGING, LAUGHING, CRYING, SIGHING, AND YAWNING.

BY HARRY CAMPBELL, M.D., F.R.C.P. LoND.,

PHYSICIAN, NORTH-WEST LONDON HOSPITAL.

THE respiratory movements have wide-reaching effects. They not only lead to the flow of air to and from the lungs, but they profoundly influence the circulation of the blood and lymph ; they also affect the functions of the abdominal and pelvic viscera by rhythmically compressing and dislocating them. Now, these movements are liable to constant modification in the physiological acts of talking, shouting, singing, laughing, crying, sighing, and yawning (as also in the occasional and semi-pathological acts of sneezing, coughing, vomiting, and hiccoughing), and it therefore follows that these acts are more far-reaching in their effects than would at first sight appear, and bence are worthy of our careful study. This will the more readily be granted when it is added that they affect the body, not only by modifying the respiratory movements and thus producing the effects already mentioned, but by involving the expenditure of a considerable amount of neuro-muscular energy, and by inducing definite psychic phenomena which themselves have their physical accompaniments. In brief, any one of these acts-e.g , singing-causes (a) a modification in the circulation of blood and lymph; $(b)$ an alteration in the functions of the abdominal and pelvic viscera; and further leads to $(c)$ a considerable discharge of nervous and muscular energy; and to $(d)$ numerous changes (muscular, glandular, and other) throughout the body in consequence of the attendant psychic change.

Seeing, then, how far-reaching are the effects of these several acts, and remembering how large a part they play in normal life, we may safely conclude that they influence the functions of the body beneficially, and that an undue interf $t$ rence with them is injurious. One is apt to forget how strong is the instinct to shout and sing laugh and cry. It is especially noticeable in the savage and in the child. If these instincts are unduly repressed in the child he is sure to suffer. Crying should certainly be restrained within limits, but there can be no doubt that it is primarily physiological, not only favouring the proper expansion of the lungs and accelerating the circulation, but deadening the effects of pain and relieving nerve tension (especially in woman). Rosbach thinks it not improbable that many evils which manifest themselves in later life, such as chlorosis, contracted chest, and the phthisical habit, "may take their origin in the practice of mothers to stop their infants from screaming by soothing them to sleep in their arms or by stupefying rocking in the cradle." It is well known that children show a strong instinct to chatter and sing the first thing in the morning, and it should be allowed full vent as far as is practicable. The shouting which young people indulge in during their play is quite remarkable and is manifestly physiological The same tendency to shout is observed in young adults, especially among the poorer classes in holiday time. Though from the physiological point of view justifiable, and even beneficial, the noises they make are certiinly not always pleasing, especially to the sensitive nerves of the cultured, amongst whom this instinct is consequently suppressed, though whether altogether to the gain of the individual is questionable.

The various acts referred to will now be considered individually.

Talling.-As regards the psychic aspect of talking, thought becomes much more vivid if it finds expression, whether in speech, writing, music, or artistic production, than if it remains unexpressed. The physical effects of thought are more pronounced in talking than in writing. The cortical nervous discharges underlying it send a stream of energy towards the muscles involved in speech and gesture, and both voice and gesture can be modified to convey subtle shades of thought and feeling which cannot find expression in writing. The very expression of these refinements enhances the vividness and intensity of mentation. Talking is for this reason stimulating, and its influence in 
this respect is in a measure proportional to the gesture accompanying it. Few things are more calculated to stimulate the body, to rouse it from lethargy, than "animated" conversation. In talking, as in laughing, shouting, singing, and crying, inspiration is short, while expiration is prolonged, the exit of air being checked partly by obstruction in the glottis and partly, perhaps, by the action of the inspiratory muscles. The actual amount of work done in talking is far more than might at first sight be supposed, and should always be taken into account in reckoning $\mathrm{up}$ the quantity of exercise taken during the day. The amount of talking done by barristers, politicians, and others enables them to dispense largely with exercise as ordinarily understood; for not only do they in this way expend a considerable amount of muscular energy, but they experience the manifold advantages of active respiratory movements continued for long periods together; indeed, I believe talking to be distinctly conducive to longevity. That talking involves a considerable expenditure of energy is shown by the exhaustion which it induces in those who are nervously run down. Such are often greatly exhausted, even after a moderate day's talking. This exhaustion is due to mental as well as to muscular expenditure; indeed, in the very neurasthenic the bare process of thought may be an effort and the mere effort to think may alone cause exhaustion; and if such is the case, how much more likely is the putting of thought into speech to do so, seeing that, a part from the muscular expenditure involved in speech, thought is so much more intense when spoken than when unexpressed. Talking is a beneficial exercise in heart disease, especially in those forms in which the blood tends to be dammed back upon the lungs. The good effect is here doubtless due to the increased amplitude of the respiratory movements and to the help thus afforded to the pulmonary circulation. It is for this reason that I always encourage talking in those suffering from passive engorgement of the lungs. "The breathlessness due to dilatation of the heart," observes Sir William Broadbent, "is often relieved by exercise of the voice. I have met with numerous instances in which a clergyman has climbed into the pulpit with the utmost difficulty, and has not only preached a sermon comfortably, but has been all the better for it." 2 The good result, I take it, in these cases is attributable to the deep inspirations required by the loud voice necessary to fill a large building.

Shouting.-The psychic accompaniment of shouting is essentially emotional. Emotion is not only expressed, but sustained, and, indeed, intensified by it. Thus the shouting of children at play, itself the outcome of exuberant emotion and pent-up neuro-muscular energy, enhances the emotional outburst. In like manner the hurrabs of an applauding multitude, the cry of the huntsman, the war-whoop of the savage, the yells of an attacking force, may so exalt emotionality as to induce a condition bordering on ecstacy. A further effect of shouting is to dull sensibility, the emotional exaltation which it provokes, and the voluminous discharge of neuro-muscular energy accompanying it, inducing a corresponding depression in the sensorial sphere. It is on this principle that groaning, and still more the shriek of acute agony, bring relief. The mere sound produces a similar effect by violently energising the acoustic centres. $^{3}$ The shouting and gesticulation which accompany an outburst of passion act physiologically by relieving nerve tension; and, indeed, as Hughlings Jackson has suggested, swearing may not be without its physiological justification. Passionate outbursts are generally succeeded by a period of good behaviour, and, it may be, improved health. One frequently notices this in children, and I have also observed it in the adult. It is possible that the outbursts of irritability observed in disease, as, for instance, in gout, have their physiological as well as their pathological aspect. As regards the modifications in the respiratory movements caused by shouting the important practical point to notice is that they are increased in depth. Hence shouting favours the development of the lungs and accelerates the circulation of blood and lymph.

singing.--Singing, like shouting, is more emotional than intellectual, the degree of emotion called forth depending upon the extent to which the individual throws himself into the spirit of the song. The nature of the attendant emotion varies of course considerably, and there is a corresponding

2 The LaNCet, April 4th, 1891, p. 798.

3 A famous quack extracts his patient's teeth to the blare of trumpets and the boom of the big drum. variability in its physical correlatives : if the theme of the song be joyous the proper rendering of it is highly stimulating. In singing there is a great disproportion between inspiration and expiration, the former being much the shorter. Moreover, during these long expirations the glottis is constricted, and the free egress of air being thus prerented intra-pulmonary air tension rises and frequently the flow of blood to and from the right heart is impeded, but this temporary retardation of the blood-flow may be more than compounded for by the acceleration which takes place during the succeeding deep inspiration. The obstruction of the glottis increases as the scale is ascended, and seeing that the expiratory force employed in the delivery of the upper notes is, for the most part, greater than that employed in the production of the lower, it follows that high singing, especially if fortissimo, impedes the circulation more than low singing. From the medical standpoint singing is a most important exercise, both by virtue of its influence on the emotions, on the respiratory movements, and on the development of the lungs. The good average health enjoyed by professional singers is in large measure attributable to the mere exercise of their calling. ${ }^{4}$ Such therapeatic importance do I attach to singing that I recommend it wherever opportunity affords. It is especially useful in defective chest development and in chronic heart disease. Oertel speaks enthusiastically of the beneficial influence of singing on the general health, and especially on the lungs, and he refers to the fact that almost all eminent singing masters can tell of serious cases of lung disease which have been cured by their method of singing. He thinks there can be no doubt that weak chests of various kinds can be greatly improved by it, and he would even appear to include phthisis. "In consequence of the reports sent in from various quarters on the healthy influence of singing on the respiration and circulation and on the strengthening and nutrition of the lung, the practice of singing has been introduced even into prisons in order to antagonise pulmonary consumption, which generally develops in a short time amongst the convicts. The method of singing devised by Fried-Grell is especially adapted for our national schools and ought to be generally introdaced, like gymnastics, from a sanitary point of view." 5

Laughter.-The psychic accompaniment of laughter being joyous emotion, its effect is stimulating, and it has been truly said that the man who makes us laugh is a public benefactor. Its beneficial effect on the body is illustrated by the saying, "Laugh and grow fat." The expiratory act in laughter is greatly prolonged, and, the glottis being partly closed, intra-pulmonary tension is increased; and thus in excessive laughter there may be considerable impediment to the flow of blood through the lungs, as shown by the turgid head and neck. This disadvantage-for in most cases of heart disease it is a disadvantage - is far more than compensated for by other effects, foremost among which must be reckoned the deep inspirations which separate the individual paroxysms.

Crying.-In thinking of the term "crying" one must distinguish between the mere shedding of tears, and weeping accompanied by sobbing. In the one the effects are limited, while in the other the entire body may be convulsed, I have already referred to the beneficial effects of crying in children. The crying of the infant is peculiar. Expirations are prolonged sometimes for as much as half a minute, and are interrupted by short inspirations. During the expirations the glottis is contracted and the intra-pulmonary pressure rises considerably. Not only is the pulmonary circulation thereby greatly impeded, as shown by the swollen veins of the head and neck, but bronchial mucus, flatus, and other noxious matters are evacuated. The paroxysm is succeeded by rapid deep respirations, which restore the equilibrium of the circulation. Women likewise often derive benefit from " a good cry"-the profuse flow of tears lessens blood-pressure within the cranium; the voluminous discharge of nerve energy relieves nerve tension; the sobbing movements of respiration influence in a very decided and doubtless beneficial way the circulation and the movements of the abdomino-pelvic viscera; while the widespread con traction of the muscle system has probably also a good effect

4 The splendid chest development of public singers is, of course, no entirely attributable to the constant exercise of the voice, since no on can attain a high excellence without having a good chest developmen in the first instance. It must also be observed that every singer who attains to fame is careful to lead a healthy life.

3 Von Ziemssen's General Therapeutics, p. 582 
How pronounced are the dynamic effects induced by completely abandoning oneself to a fit of crying is shown by the exhaustion which it entails. It is partly tbrough this exhaustion that crying induces sleep; we hear of "crying oneself to sleep," though this must be but a very crude explanation of the phenomenon. The tendency of women to cry should, of course, be kept within proper bounds, but certainly harm may result from its complete suppression, as Tennyson recognises in the line-

$$
\text { "She must weep or she will die." }
$$

It is said that women who are able to find relief in tears keep their youth longer than those who repress them. The internal cankering action "like a worm $i$ ' the bud" of pent-up emotion is not only a beautiful poetic conceit, but a profound physiological truth. In short, strong emotion should receive expression- " give sorrow words."

Sighing.- "A sigh is a deep thoracic respiration, with retraction of the abdomen." ${ }^{B}$ The retraction of the abdominal muscles leads to a compression of the splanchnic veins. This compression is probably increased by slight descent of the diaphragm. The blood is thus pressed out of those veins into the right heart, and the flow into this chamber is further favoured by the deep inspiration which also aids the circulation through the lungs. Now Hill has pointed out 7 that the blood accumulates in the splanchnic veins in syncope, and that "a deep sigh is the first obvious sign of improvement." That the act of sighing in syncope has the effect maintained is shown by the observation of Stephen Hales. He noticed that the arterial blood-pressure in horses rises considerably on deep sighing and that the same effect is produced in dogs by firmly pressing the abdomen. ${ }^{8}$ The sigh of syncope is comparatively rare. A much more common cause of sighing I believe to be shallow breathing, however induced. Thus sadness and a sense of weariness or boredom are wont to be attended by shallow breathing, and in all of them sighing is frequent. In consequence of this shallow breathing blood-aeration lags behind, and the blood tends to accumulate in the right heart and systemic veins. The sigh benefits by promoting the aeration of the blood and quickening the pulmonary circulation, and it is for similar reasons that sighing is apt to occur during a state of "breathless attention" - when the attention, i.e., is so strained that one forgets, as it were, to breathe adequately.

Yanning.-It is difficult to describe accurately this act. A deep and prolonged inspiration is taken through the mouth and nose, the mouth being widely opened and the nares dilated. The opening of the mouth is effected by a strong tonic contraction of the depressors of the lower jaw and by the extension of the head and consequent elevation of the upper jaw. At the same time the limbs and trunk are "stretched." There is, in short, a widespread tonic contraction of the rauscle system. This continues and indeed reaches its climax during the expiratory portion of the act, the mouth being kept open till towards the end of it The expiratory blast is directed solely through the mouth owing to the elevation of the soft palate, and the vocal cords approximate, a characteristic sound being produced in the larynx, while there is a peculiar contraction of the muscles at the back of the throat the nature of which I do not understand. There can be little doubt that one of the objects of rawning is the exercise of muscles which have been for a long time quiescent, and the acceleration of the blood and lymph flow which has in consequence of this quiescence become sluggish. Hence its frequency after one has remained for some time in the same position-e.g., when waking in the morning. Coopperating with this cause is wleepiness and the shallow breathing which it entails. This factor, as well as muscle-quiescence, is apt to attend the sense of boredom which one experiences in listening to a dull sermon. Hence it is that the bored individual is apt to jawn. As in the case of sighing, the deep breath which accompanies the act of yawning compensates for the shallow breathing which is so apt to excite it. I am unable to offer any explanation of the yawning-or, rather, gaping-which is induced by exhaustion from want of fooù. This mere gaping is, however, quite distinct from yawning, which is a much more complex phenomenon. Allied to yawning is a modification of the breathing which is apt to occur during sleep. A deep breath is taken more or less suddenly, and this is

6 I. Hill: Journal of Physiology, rol. xv,, p. 48.

8 Statist Essays (London, 1733), rol. ii., p 33, referred to by L. Hill, op. cit. followed by a long-drawn out expiration with probably closed glottis and a groaring sound. This kind of breathing bas certainly some physiological import, but what it is I cannot say.

Wimpole-street, $\mathrm{W}$.

\section{AN UNUSUAL CASE OF PARALYSIS OF THE OCULAR MUSCLES.}

By LESLIE BUChaNAN, M.B. GlasG,

PATHOLOGIST AND CLINICAL ASSISTANT, GLASGOW EYE INFIRMaRY.

THE patient who was the subject of this unusual train of symptoms came to me first on Oct. 31st, 1894, complaining of headache, giddiness, and difficulty of vision, and was accompanied by her sister whose support was necessary to enable her to walk at all correctly. The history of her condition was that headache was the first evidence of any disorder and appeared just thirteen days before being closely followed by giddiness, and that difficulty of vision was the last symptom to appear. The past history of the patient was practically withont blemish, she not having had any serious or prolonged illness in her life. There was no evidence of gout, rheumatism, syphilis tuberculosis, or, indeed, of any dyscrasia, and the patient was not at any time either neurotic or hysterical. There was no history of any injury at all likely to give rise to such symptoms as the patient complained of. The family history of the patient was of the very soundest description, no bodily habit or tendency in any way prejudicial being transmitted, so far as could be found. There had not been any nervous disease in the family. When first seen, the patient, who was about thirty years of age, was, apart from the aforementioned symptoms, in perfect health. neither anæmic nor plethoric, although well nourished; and further she was of sober manner and quiet demeanour. She stated that her headaches were almost entirely frontal, not tending to one side more than the other, and that they generally began with pain in the eyes. They were, further, distinctly worse after using the eyes for a time, ceasing at night, but often being present on waking in the morning, more especially if she had been working at her employmenti.e., dressmaking-the previous night. The vertigo was of a general nature, and there was no inclination to fall to one side more than the other. The patient could stand well with the eyes closed, the feet together, and the hands raised high above the head. She could not "walk a line" at all well, and any effort to hold on to or grasp anything evidently increased the difficulty of the process, which seemed to indicate that visual effort was--in part at leastthe cause of the deficiency. The visual disturbance appeared to be difficult to define as it tended in no very special direction but resulted, somehow, in confusion (the patient had never noticed that she saw objects doubled until, by the usual methods, it was pointed out to her). It was manifest, on examination, that there was a nearly complete paralysis of the external rectus muscle of the right eye, this muscle being unable to turn the eye outwards further than the middle line, there being, however, no convergent strabismus at this date. Diplopia was, of course, homonymous and was present even when an object was held $15^{\circ}$ to the left of the middle line at $10 \mathrm{ft}$. distance. Ophthalmoscopic examination revealed neither error of refraction nor change in any degree of the fundus of either eye, and there was no evidence of internal ophthalmoplegia. The fields were normal both for colour and white light. The knee and other reflexes were examined, but no evidence of nervons affection was found in any part. Examination of the general organs proved to be entirely negative, and inquiry failed to elicit evidence of any functional inaccuracy or disorder; indeed, there appeared to be absolutely no signs, other than those before mentioned, of any disease. It seemed highly probable, then, that both headaches and vertigo were the results rather than the companion symptoms of the paralysis. A guarded prognosis was given, complete ocular rest was ordered, and the use for a prolonged period recommended of a mixture containing the iodide and bromide of potassium with nux vomica. As regards the course of the disease it was found to be moderately rapid, for on Nov. 14th the paralysis was complete, there being 